\title{
Effects of Tourniquet Application on Faster Recovery after Surgery and Ischemia-Reperfusion Post-Total Knee Arthroplasty, Cementation through Closure versus Full-Course and Nontourniquet Group
}

\author{
Qinggang Cao, $\mathrm{MD}^{1, *(-)}$ Qiong $\mathrm{Wu}, \mathrm{MD}^{2, *} \quad$ Yun Liu, $\mathrm{MD}^{3, *}$ Zhiwei He, PhD ${ }^{1, *} \quad$ Yu Cong, PhD ${ }^{1, *}$ \\ Jia Meng, $\mathrm{PhD}^{1}$ jianning Zhao, $\mathrm{PhD}^{1,3}$ Nirong Bao, $\mathrm{PhD}^{1,3}$
}

1 Department of Orthopaedic Surgery, Jinling Hospital, Nanjing, China
2 Department of Scientific Research and Training, Division of Health
Service, General Hospital of Eastern theater of People's Liberation
Army, Nanjing, Jiangsu, China
${ }^{3}$ Health Technology Cadre Training, Jingling Hospital, Nanjing, China

Address for correspondence Nirong Bao, PhD, Department of Orthopaedic Surgery, Jinling Hospital, Nanjing, 210002, China (e-mail: bnrbnr@sina.com).

J Knee Surg 2022;35:1577-1586.

\begin{abstract}
Pneumatic tourniquets are used in total knee arthroplasty (TKA) for surgical field visualization and improved cementation; however, their use is controversial. This study aimed to assess the effects of tourniquet application on faster recovery post-TKA. Our hypothesis was that inflammation and limb function would be similar with different tourniquet applications. A prospective randomized double-blinded trial assessed tourniquets effects on postoperative pain, swelling, and early outcome in TKA. In present study, 50 TKAs were enrolled in each group as follows: full course (FC), cementation through closure (CTC), and no tourniquet (NT), CTC as treatment group while FC and NT as control groups. Topical blood samples of $3 \mathrm{~mL}$ from the joint cavity and drainage bags were obtained at special time point. At last, all samples such as tumor necrosis factor-a (TNF-a), C-C motif chemokine ligand 2 (CCL2), pentraxin 3 (PTX3), prostaglandin E2 (PGE2), superoxide dismutase 1 (SOD1), and myoglobin (Mb) were detected by ELISA. Active and passive range of motion (ROM) values, pain score by the visual analog scale (VAS), change of thigh circumference were recorded at special time point as well. In topical blood, the change of inflammatory factors, such as TNF-a, PTX3, CCL2, PGE2, SOD1, and Mb, was lower in CTC and NT groups than in FC group

Keywords

- tourniquet

- total knee arthroplasty

- swelling

- pain $(p<0.01$ and 0.05$)$. Although VAS and ROM were comparable preoperatively in three groups $(p>0.05)$, the perimeter growth rate was lower, pain scores (VAS) were reduced, and ROM values were improved in CTC and NT groups compared with FC group at T4, T5, and T6 postoperatively $(p<0.01$ and 0.05$)$. Improved therapeutic outcome was observed in the CTC group, indicating patients should routinely undergo TKA with cementation through closure tourniquet application.
\end{abstract}

Co-first authors.

received

August 10, 2018

accepted after revision

March 12, 2021

article published online

May 5, 2021 (c) 2021. Thieme. All rights reserved.

Thieme Medical Publishers, Inc., 333 Seventh Avenue, 18th Floor, New York, NY 10001, USA
DOI https://doi.org/

10.1055/s-0041-1728814. ISSN 1538-8506. 
With advances in technology and instruments, total knee arthroplasty (TKA) is currently considered one of the most effective orthopaedic surgeries. ${ }^{1}$ A tourniquet can provide a bloodless field of view for surgery, may help reduce intraoperative blood loss and improve cement penetration, and could also shorten the operation time. However, tourniquet use can be associated with postoperative swelling, joint stiffness, muscle injury or nerve dysfunction, and infrequent major nerve or vascular injury. Moreover, some studies found that the use of a tourniquet may increase postoperative hidden blood loss. ${ }^{2-9}$

The most common complications related to the use of tourniquet are seen in early postoperative period and therefore these may have short-term effects on the patient. ${ }^{10,11}$ It was believed that tourniquet duration is a very important determinant of faster recovery after operation. There is still no consensus regarding the duration of its use during TKA. Our prospective randomized controlled trial (RCT) was aimed to evaluate the outcomes of short duration versus long duration and no tourniquet (NT) during TKA. We hypothesized that inflammation and limb function would be similar with different tourniquet applications. Others will take the information and decide if this will change how they use tourniquets.

\section{Patients and Methods}

After obtaining institutional ethics committee approval and patient consent, 150 patients with unilateral knee osteoarthritis were randomized into three groups for TKA (50/group), CTC as treatment group while full course (FC) and NT as control group. Inclusion and exclusion criteria were predefined (-Table 1). Patients with symptomatic peripheral vascular disease or contraindication to tourniquet use were excluded. The consolidated standards for reporting trials are summarized in the study flow chart (-Fig. 1). The patient demographics showed no significant differences.

All patients underwent TKA by a single surgeon using a medial parapatellar approach and standardized technique using a cemented, fixed bearing, posterior cruciate-retaining prosthesis with patellar resurfacing and underwent general

Table 1 Study inclusion and exclusion criteria

\begin{tabular}{|l|l|}
\hline Inclusion criteria & Exclusion criteria \\
\hline $\begin{array}{l}\text { Symptomatic grade IV } \\
\text { knee OA }\end{array}$ & $\begin{array}{l}\text { Any hematological disease } \\
\text { (e.g., coagulopathy) }\end{array}$ \\
\hline $\begin{array}{l}\text { Signed informed consent } \\
\text { for the procedure }\end{array}$ & Any infective foci in the body \\
\hline $\begin{array}{l}\text { Unilateral TKA for } \\
\text { the first time }\end{array}$ & $\begin{array}{l}\text { A history of } \\
\text { immunosuppression }\end{array}$ \\
\hline 50 years $\leq$ age $\leq 80$ years & $\begin{array}{l}\text { Any peripheral neurovascular } \\
\text { disease }\end{array}$ \\
\hline & $\begin{array}{l}\text { Inflammatory arthritis, } \\
\text { like rheumatoid arthritis }\end{array}$ \\
\hline
\end{tabular}

Abbreviations: IV, intravenous; OA, osteoarthritis; TKA, total knee arthroplasty. anesthesia without regional blocks or local anesthesia in an effort to rule out confounding factors that may influence pain scores.

In present study, an above knee tourniquet (width, $10.5 \mathrm{~cm}$; length, $65.5 \mathrm{~cm}$; VBM Medizintechnik GmbH, Sulz, Germany) was used for patients in CTC or FC group and NT group during surgery. The tourniquet was inflated to $280 \mathrm{~mm}$ $\mathrm{Hg}$ just prior to initial skin incision in FC group and to $280 \mathrm{~mm} \mathrm{Hg}$ immediately prior to mounting the cemented prosthesis in the CTC group. The tourniquet was deflated following wound closure and application of dressings, while NT applied throughout entire surgery for patients in the NT group. The average tourniquet duration and operation time were 37.5/70.2 minutes in CTC group, 66.4/66.4 minutes in FC group and 0/78.4 minutes in NT group.

Intra-articular drainage on low suction was performed prior to wound closure and removed on day 2 postoperatively. Topical blood samples of $3 \mathrm{~mL}$ from the joint cavity (T1) and drainage bags at T2, T3, and T5 in all cases were obtained. Plasma was separated by centrifugation at 3,000 rpm and $4^{\circ} \mathrm{C}$ for 10 minutes. Then, the supernatant was stored at $-80^{\circ} \mathrm{C}$ for further analysis. Serum concentrations of tumor necrosis factor-a (TNF-a), C-C motif chemokine ligand 2 (CCL2), pentraxin 3 (PTX3), and prostaglandin E2 (PGE2) were measured with a specific human ELISA kit (Fcmacs, Nanjing, China). Superoxide dismutase 1 (SOD1) and myoglobin (Mb) were determined with another human ELISA kit (Jiangsu KeyGEN BioTECH Corp. Ltd.). All operations were performed according to the manufacturers' instructions.

The same standardized physiotherapy protocol was used in all patients postoperatively. Active and passive range of motion (ROM) values were evaluated. Pain score was measured by the visual analog scale (VAS). Change of thigh circumference was recorded using a measuring tape at $10 \mathrm{~cm}$ above the superior pole of the patella. VAS, ROM, and the change of thigh circumference were recorded before operation (T0) and at 12 hours (T4), 24 hours (T5), and 48 hours (T6) after tourniquet removal, respectively.

Electrocautery was used for hemostasis. Intraoperative blood loss was measured (volume in suction container amount of saline wash used) + (total weight of wet sponges used - total weight of dry sponges used) in all cases. Postoperative blood loss was determined from closed suction drain output. On the second postoperative day, hemoglobin assessment and of the knees were performed. The patients were mobilized on day 1 postoperatively and discharged when they were safe for independent ambulation. The primary outcome measures included inflammatory factors such as TNF-a, CCL2, PTX3, PGE2, SOD1, and Mb, as well as clinical function indicators such as VAS, ROM, and change of thigh circumference. Secondary outcome measures included blood loss, transfusion requirements, and hospitalization days. There were no complications directly related to tourniquet application in this study.

\section{Randomization}

A surgeon not involved in the study removed slips from nontransparent sealed envelopes. The knees selected for surgery were randomly allocated to the CTC or FC or NT 


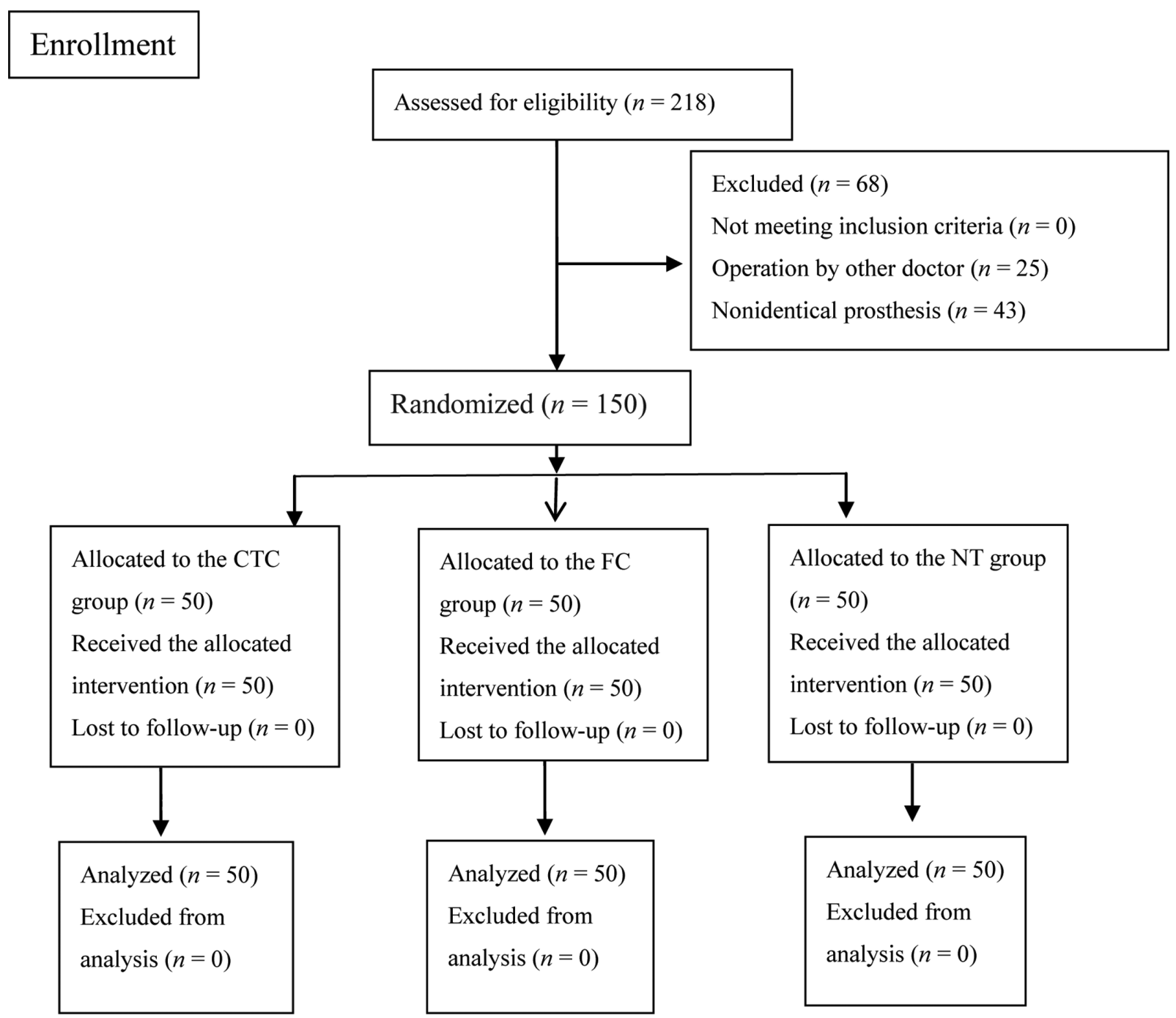

Fig. 1 Consolidated standards of reporting trials (CONSORT) flow diagram for the study. CTC, cementation through closure; FC, full course; NT, no tourniquet.

group (50/group). All eligibility criteria (-Table 1) were assessed and all patients were evaluated for 2 weeks, with no patients lost to follow-up.

\section{Blinding}

Patients and the investigator collecting data were blinded during the procedure and postoperative follow-up.

\section{Statistical Analysis}

Analysis of variance (ANOVA) and $x^{2}$-test were used for statistical analysis, and $p<0.05$ was considered statistically significant. The SPSS Statistics 21.0 software (SPSS, Chicago, IL) was used for data analysis.

\section{Results}

Change in distal thigh circumference was compared in three groups. Between groups: CTC and FC ( $p=0.026$ at T4, $p<0.01$ at T5, and $p=0.003$ at T6), NT, and FC ( $p=0.014$ at T4, $p<0.01$ at T5, and $p=0.021$ at T6), while between CTC and
NT groups, there was no statistical difference ( $p>0.05$ at T2, T3, and T4; - Fig. 2A; - Table 2).

Pain scores (VAS) were compared in three groups. Between groups: CTC and FC ( $p<0.01$ at T4, T5, and T6), NT and FC ( $p<0.01$ at T4, T5, and T6), CTC and NT ( $p>0.05$ at T4, T5, andT6), while between groups: CTC and FC, NT and FC, NT and CTC ( $p>0.05$ at T0; - Fig. 2B; - Table 3 ).

ROM values were compared in three groups. Between groups: CTC and FC ( $p<0.01$ at T4, T5, and T6), NT and FC ( $p=0.008$ at T4, $p=0.019$ at T5, and $p=0.03$ at T6), and CTC and NT ( $p=0.002$ at T4, $p<0.01$ at T5 and T6), while there were no differences compared between the three groups at T0 ( $p>0.05$; - Fig. 2 C; - Table 4).

In topical blood, there were no significant differences statistically in TNF-a, PTX3, CCL2, SOD1, PGE2, and Mb amounts between the three groups at $\mathrm{T} 1$, respectively ( $p>0.05$, - Fig. 3 ). However, there were statistically significant differences in the three groups at T2, T3, and T5.

TNF-a: between groups: CTC and FC $(p<0.01$ at T2, T3, $p=0.01$ at T5), NT and FC ( $p<0.01$ at T2, T3, and T5), and CTC 

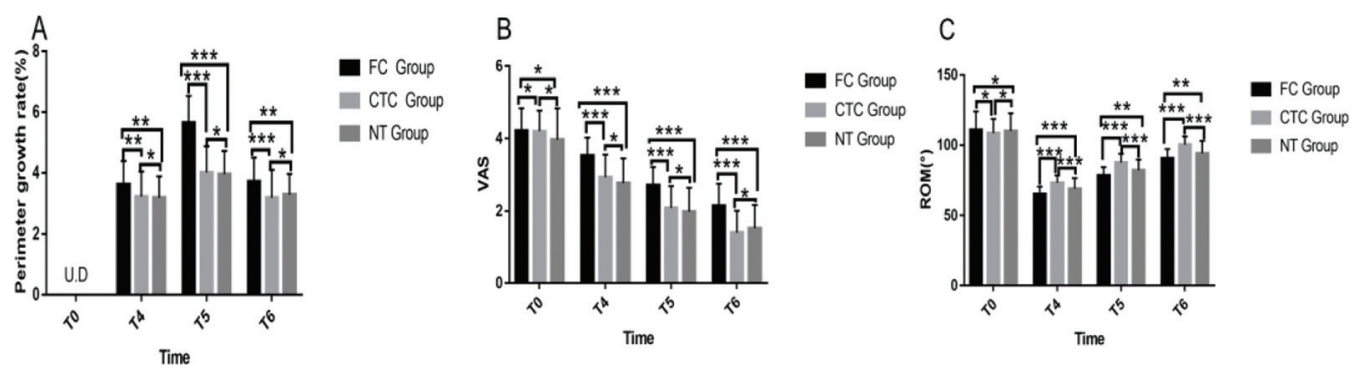

Fig. 2 (A) Perimeter growth rate, (B) VAS, and (C) ROM in FC group, CTC group, and NT group. Data are mean \pm standard deviation (SD) • > 0.05 $0.01 \leq^{* *} \leq 0.05,{ }^{* * *}<0.01$. CTC, cementation through closure; FC, full course; NT, no tourniquet; ROM, range of motion; VAS, visual analog scale.

Table 2 Comparison of changes in distal thigh circumference

\begin{tabular}{|l|l|l|l|l|}
\hline Group & Cases & T4 & T5 & T6 \\
\hline CTC group & 50 & $3.22 \pm 0.82$ & $4.08 \pm 0.86$ & $3.19 \pm 0.9$ \\
\hline FC group & 50 & $3.62 \pm 0.77$ & $5.65 \pm 0.89$ & $3.72 \pm 0.31$ \\
\hline NT group & 50 & $3.19 \pm 0.69$ & $3.95 \pm 0.77$ & $3.28 \pm 0.68$ \\
\hline CTC vs. FC & & $p=0.026$ & $p<0.01$ & $p=0.003$ \\
\hline CTC vs. NT & & $p=0.633$ & $p=0.214$ & $p=0.351$ \\
\hline NT vs. FC & & $p=0.014$ & $p<0.01$ & $p=0.021$ \\
\hline
\end{tabular}

Abbreviations: CTC, cementation through closure; FC, full course; NT, no tourniquet.

Table 3 Comparison of pain scores (VAS)

\begin{tabular}{|l|l|l|l|l|}
\hline Group & Cases & T4 & T5 & T6 \\
\hline CTC group & 50 & $2.92 \pm 0.63$ & $2.08 \pm 0.61$ & $1.4 \pm 0.61$ \\
\hline FC group & 50 & $3.52 \pm 0.53$ & $2.7 \pm 0.51$ & $2.14 \pm 0.6$ \\
\hline NT group & 50 & $2.76 \pm 0.69$ & $1.98 \pm 0.65$ & $1.52 \pm 0.65$ \\
\hline CTC vs. FC & & $p<0.01$ & $p<0.01$ & $p<0.01$ \\
\hline CTC vs. NT & & $p=0.272$ & $p=0.146$ & $p=0.211$ \\
\hline NT vs. FC & & $p<0.01$ & $p<0.01$ & $p<0.01$ \\
\hline
\end{tabular}

Abbreviations: CTC, cementation through closure; FC, full course; NT, no tourniquet; VAS, visual analog scale.

Table 4 Comparison of ROM values

\begin{tabular}{|l|l|l|l|l|}
\hline Group & Cases & T4 & T5 & T6 \\
\hline CTC group & 50 & $73.26 \pm 5.45$ & $87.62 \pm 6.19$ & $100.32 \pm 5.88$ \\
\hline FC group & 50 & $65.42 \pm 5.25$ & $78.44 \pm 5.87$ & $90.5 \pm 6.31$ \\
\hline NT group & 50 & $68.78 \pm 7.69$ & $82.06 \pm 7.71$ & $94.22 \pm 8.68$ \\
\hline CTC vs. FC & & $p<0.01$ & $p<0.01$ & $p<0.01$ \\
\hline CTC vs. NT & & $p=0.002$ & $p<0.01$ & $p<0.01$ \\
\hline NT vs. FC & & $p=0.008$ & $p=0.019$ & $p=0.03$ \\
\hline
\end{tabular}

Abbreviations: CTC, cementation through closure; FC, full course; NT, no tourniquet; ROM, range of motion.

and NT $(p<0.01$ at T2, T5, $p=0.311$ at T3; - Fig. 3A; -Table 5).

PTX3: between groups: CTC and FC $(p=0.021$ at T2, $p<0.01$ at T3, T5), NT and FC ( $p=0.012$ at T2, $p<0.01$ at T3, T5), and CTC and NT ( $p>0.05$ at T2, T3, and T5; - Fig. 3B; -Table 6).
CCL2: between groups: CTC and FC $(p<0.01$ at T2, T3, and T5), NT and FC ( $p<0.01$ at T2, T3, and T5), and CTC and NT $(p<0.05$ at T2, T3, and T5; - Fig. 3C; - Table 7$)$.

SOD1: between groups: CTC and FC ( $p<0.01$ at T2, T3, and T5), NT and FC ( $p=0.02$ at T2, $p<0.01$ at T3 and T5), and CTC and NT ( $p<0.05$ at T2, T3, and T5; - Fig. 3D; - Table 8).

PGE2: between groups CTC and FC $(p<0.01$ at T2, T3, and T5), NT and FC ( $p<0.01$ at T2, T3, and T5), and CTC and NT $(p<0.05$ at T2, T3, and T5; - Fig. 3E; - Table 9).

Myoglobin: between groups CTC and FC $(p<0.01$ at T2, T3, and T5), NT and FC ( $p<0.01$ at T2, T3, and T5), and CTC and NT $(p<0.05$ at T2, T3, and T5; - Fig. 3F; - Table 10).

In addition, intraoperative blood loss between groups CTC and FC $(p<0.01)$, CTC and NT $(p<0.01)$, NT and FC $(p<0.01)$. Postoperative blood loss between groups CTC and FC $(p<0.01)$, CTC and NT $(p<0.01)$. Total blood loss (intraoperative + postoperative) between groups CTC and NT $(p<0.01)$ and NT and FC $(p<0.01 ;-$ Table 11).

Blood transfusion, the threshold of postoperative blood transfusion was hemoglobin $<8 \mathrm{~g} / \mathrm{dL}$. Five of 50 patients in FC group, 2 of 50 in CTC group, and 14 of 50 in NT group. Days to discharge between groups CTC and FC $(p<0.05)$, CTC and NT $(p<0.05)$, and NT and FC $(p>0.05)$. There were no events of thromboembolism and deep vein thrombosis in every group (-Table 12).

\section{Discussion}

It was believed that tourniquet duration is a very important determinant of faster recovery after operation. The most common complications related to the use of tourniquet are seen in early postoperative period and therefore these may have short-term effects on the patient. ${ }^{10,11}$ There is still no consensus regarding the duration of its use during TKA.

Studies showed that patients who have had tourniquets applied have lower functional scores due to quadriceps weakness after surgery, as well as residual pain in the thigh. ${ }^{12-17}$ Huang et al ${ }^{18}$ demonstrated that using tourniquet full-time causes more excessive inflammation and muscle damage. Zhang et $\mathrm{al}^{19}$ reported that the tourniquet use negatively affects the early postoperative rehabilitation as well. Therefore, in recent years, orthopaedic surgeons tend to perform operations without a tourniquet or only use one with a cement application. 


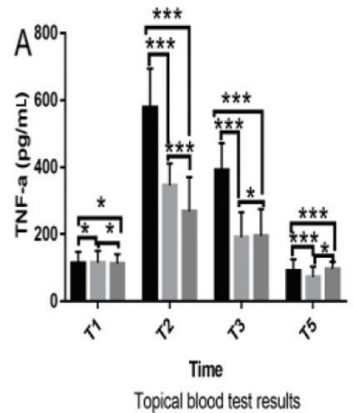

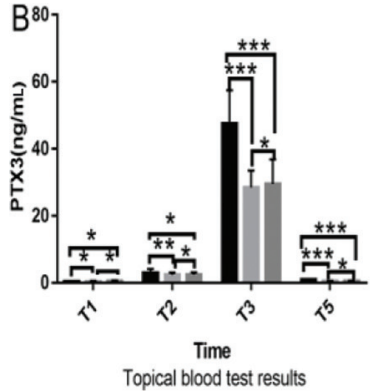

Topical blood test results
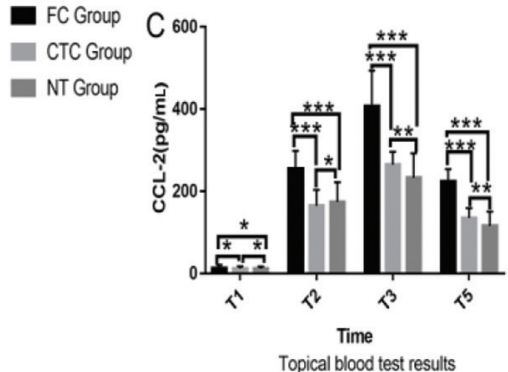

FCGroup

CTC Group

NT Group
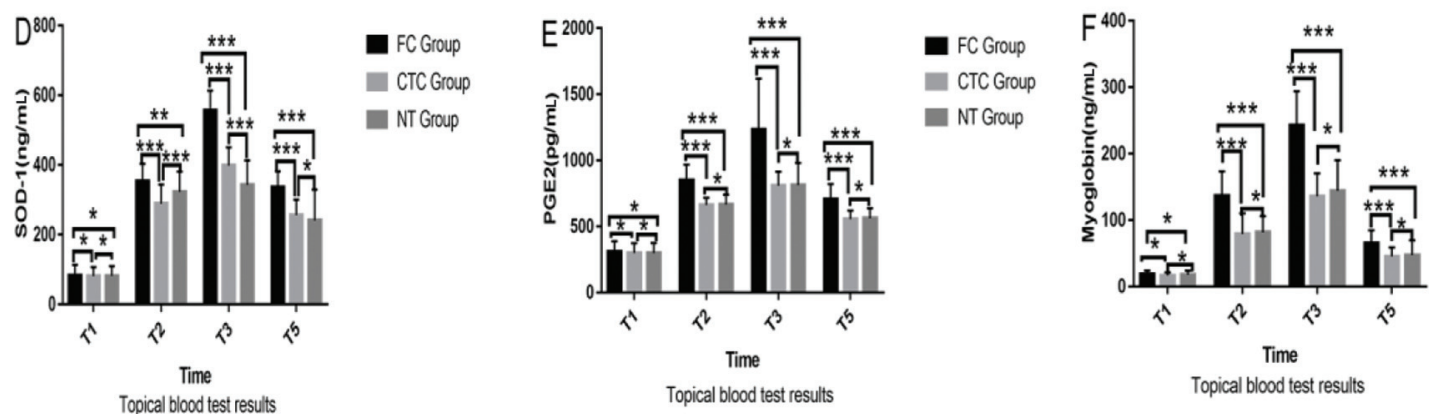

FC Group

- CTC Group

NT Group

Fig. 3 (A-F) Topical blood data for FC group, CTC group, and NT group. Data are mean \pm standard deviation (SD) *>0.05 $0.01 \leq^{* *} \leq 0.05{ }^{* * *}<$ 0.01. CCL1, C-C motif chemokine ligand 2; CTC, cementation through closure; FC, full course; NT, no tourniquet; PGE2, prostaglandin E-2; PTX3, pentraxin 3; SOD1, superoxide dismutase 1; TNF-a, tumor necrosis factor.

Table 5 Comparison of TNF-a

\begin{tabular}{|l|l|l|l|l|}
\hline Group & Cases & T2 & T3 & T5 \\
\hline CTC group & 50 & $345.43 \pm 65.59$ & $190.95 \pm 77.44$ & $72.5 \pm 31.2$ \\
\hline FC group & 50 & $578.63 \pm 115.77$ & $399.6 \pm 80.71$ & $90.12 \pm 34.91$ \\
\hline NT group & 50 & $268.17 \pm 63.31$ & $195.5 \pm 80.62$ & $96.53 \pm 21.68$ \\
\hline CTC vs. FC & & $p<0.01$ & $p<0.01$ & $p=0.01$ \\
\hline CTC vs. NT & & $p<0.01$ & $p=0.311$ & $p<0.01$ \\
\hline NT vs. FC & & $p<0.01$ & $p<0.01$ & $p<0.01$ \\
\hline
\end{tabular}

Abbreviations: CTC, cementation through closure; FC, full course; NT, no tourniquet; TNF-a, tumor necrosis factor-a.

Table 6 Comparison of PTX3

\begin{tabular}{|l|l|l|l|l|}
\hline Group & Cases & T2 & T3 & T5 \\
\hline CTC group & 50 & $4.33 \pm 0.51$ & $28.42 \pm 5.14$ & $0.53 \pm 0.12$ \\
\hline FC group & 50 & $5.87 \pm 1.37$ & $47.6 \pm 9.71$ & $0.94 \pm 0.19$ \\
\hline NT group & 50 & $4.46 \pm 0.73$ & $29.45 \pm 7.62$ & $0.57 \pm 0.17$ \\
\hline CTC vs. FC & & $p=0.021$ & $p<0.01$ & $p<0.01$ \\
\hline CTC vs. NT & & $p=0.252$ & $p=0.263$ & $p=0.515$ \\
\hline NT vs. FC & & $p=0.012$ & $p<0.01$ & $p<0.01$ \\
\hline
\end{tabular}

Abbreviations: CTC, cementation through closure; FC, full course; NT, no tourniquet; PTX3, pentraxin 3.

Stroh et $\mathrm{al}^{20}$ found excellent clinical outcomes in TKA performed without a tourniquet and commented that the use of tourniquet negatively impacts patient outcome. Zhang et $\mathrm{al}^{21}$ showed small benefits in the early postoperative period in their study in surgery done without tourniquet. Li et $\mathrm{al}^{22}$ found that increased pain and swelling were found in long duration tourniquet groups during the first postoperative week and the patients undergoing TKA without tourniquet achieved earlier straight-leg raising (SLR) and knee flexion. In our RCT, at T4, T5, and T6, change in distal thigh circumference was less in NT group than in FC group, the pain control was better in NT group than in FC 
1582 Effects of Tourniquet Application on Faster Recovery after TKA Cao et al.

Table 7 Comparison of CCL2

\begin{tabular}{|l|l|l|l|l|}
\hline Group & Cases & T2 & T3 & T5 \\
\hline CTC group & 50 & $164.9 \pm 38.62$ & $268.42 \pm 31.43$ & $135.33 \pm 23.84$ \\
\hline FC group & 50 & $254.67 \pm 44.14$ & $406.92 \pm 86.61$ & $223.7 \pm 30.48$ \\
\hline NT group & 50 & $176.32 \pm 0.73$ & $232.61 \pm 60.11$ & $115.52 \pm 34.99$ \\
\hline CTC vs. FC & & $p<0.01$ & $p<0.01$ & $p<0.01$ \\
\hline CTC vs. NT & & $p=0.048$ & $p=0.034$ & $p=0.004$ \\
\hline NT vs. FC & & $p<0.01$ & $p<0.01$ & $p<0.01$ \\
\hline
\end{tabular}

Abbreviations: CCL2, C-C motif chemokine ligand 2; CTC, cementation through closure; FC, full course; NT, no tourniquet.

Table 8 Comparison of SOD1

\begin{tabular}{|l|l|l|l|l|}
\hline Group & Cases & T2 & T3 & T5 \\
\hline CTC group & 50 & $288.72 \pm 55.38$ & $398.43 \pm 52.87$ & $256.27 \pm 44.99$ \\
\hline FC group & 50 & $352.74 \pm 50.75$ & $556.42 \pm 57.06$ & $336.27 \pm 46.08$ \\
\hline NT group & 50 & $322.91 \pm 58.45$ & $343.31 \pm 70.23$ & $240.9 \pm 54.56$ \\
\hline CTC vs. FC & & $p<0.01$ & $p<0.01$ & $p<0.01$ \\
\hline CTC vs. NT & & $p=0.006$ & $p<0.01$ & $p=0.044$ \\
\hline NT vs. FC & & $p=0.02$ & $p<0.01$ & $p<0.01$ \\
\hline
\end{tabular}

Abbreviations: CTC, cementation through closure; FC, full course; NT, no tourniquet; SOD1, superoxide dismutase 1.

Table 9 Comparison of PGE2

\begin{tabular}{|l|l|l|l|l|}
\hline Group & Cases & T2 & T3 & T5 \\
\hline CTC group & 50 & $662.43 \pm 58.12$ & $809.95 \pm 105.78$ & $555.9 \pm 65.6$ \\
\hline FC group & 50 & $849.51 \pm 118.84$ & $1,234.98 \pm 281.25$ & $706.09 \pm 116.48$ \\
\hline NT group & 50 & $666.42 \pm 74.93$ & $814.18 \pm 168.91$ & $562.06 \pm 77.97$ \\
\hline CTC vs. FC & & $p<0.01$ & $p<0.01$ & $p<0.01$ \\
\hline CTC vs. NT & & $p=0.731$ & $p=0.762$ & $p=0.644$ \\
\hline NT vs. FC & & $p=0.02$ & $p<0.01$ & $p<0.01$ \\
\hline
\end{tabular}

Abbreviations: CTC, cementation through closure; FC, full course; NT, no tourniquet; PGE2, prostaglandin E2.

Table 10 Comparison of myoglobin

\begin{tabular}{|l|l|l|l|l|}
\hline Group & Cases & T2 & T3 & T5 \\
\hline CTC group & 50 & $79.11 \pm 31.52$ & $135.93 \pm 34.55$ & $43.35 \pm 13.79$ \\
\hline FC group & 50 & $136.37 \pm 37.27$ & $242.52 \pm 51.64$ & $65.35 \pm 19.4$ \\
\hline NT group & 50 & $81.82 \pm 24.27$ & $144.15 \pm 46.29$ & $47.56 \pm 22.14$ \\
\hline CTC vs. FC & & $p<0.01$ & $p<0.01$ & $p<0.01$ \\
\hline CTC vs. NT & & $p=0.706$ & $p=0.362$ & $p=0.44$ \\
\hline NT vs. FC & & $p=0.02$ & $p<0.01$ & $p<0.01$ \\
\hline
\end{tabular}

Abbreviations: CTC, cementation through closure; FC, full course; NT, no tourniquet.

group, the ROM values were better in NT group than in FC group. The result is consistent with the original conclusion (-Fig. 2; - Tables 2,3,4).

The possible reason is the direct damage of the tourniquet and reperfusion injury that might increase pain that would hamper patients' postoperative rehabilitation. ${ }^{23}$ And addi- tional limb swelling in the long-duration tourniquet group after TKA might cause an increased weight in the affected limb that is sufficient to require more muscle strength for performing SLR. Furthermore, Dennis et $\mathrm{al}^{12}$ indicated that patients who underwent TKA using a tourniquet had diminished quadriceps strength during the first 3 months after 
Table 11 Blood loss amounts (intraoperative, postoperative, and overall)

\begin{tabular}{|l|l|l|l|l|}
\hline Blood loss $(\mathrm{mL})$ & FC group $(\boldsymbol{n}=\mathbf{5 0})$ & CTC group $(\boldsymbol{n}=\mathbf{5 0})$ & NT group $(\boldsymbol{n}=\mathbf{5 0})$ & $\boldsymbol{p}$-Value \\
\hline Intraoperative & $266.4 \pm 7.2$ & $359.4 \pm 14.5$ & $429.4 \pm 21.6$ & $<0.01$ \\
\hline Postoperative & $285.3 \pm 14.6$ & $112.9 \pm 10.1$ & $209.5 \pm 13.3$ & $<0.01$ \\
\hline Overall & $551.7 \pm 11.8$ & $472.3 \pm 10.3$ & $638.9 \pm 12.7$ & $<0.01$ \\
\hline
\end{tabular}

Abbreviations: CTC, cementation through closure; FC, full course; NT, no tourniquet.

Note: Data are mean \pm standard deviation.

Table 12 Inpatient outcomes in CTC, FC, and NT groups

\begin{tabular}{|l|l|l|l|l|}
\hline Parameter & FC group $(\boldsymbol{n}=\mathbf{5 0})$ & CTC group $(\boldsymbol{n}=\mathbf{5 0})$ & NT group $(\boldsymbol{n}=\mathbf{5 0})$ & $\boldsymbol{p}$-Value \\
\hline Transfusion requirements & 5 & 2 & 14 & \\
\hline Days to discharge & $5.9 \pm 2.4$ & $3.7 \pm 1.6$ & $6.2 \pm 2.3$ & FC vs. CTC $(p=0.043)$ \\
\cline { 3 - 5 } & & & CTC vs. NT $(p=0.03)$ \\
\cline { 3 - 5 } & & & FC vs. NT $(p=0.32)$ \\
\hline
\end{tabular}

Abbreviations: CTC, cementation through closure; FC, full course; NT, no tourniquet.

TKA. Early mobilization after TKA may be delayed in the patients with quadriceps weakness.

Interestingly, the current study showed better outcome following TKA in CTC group than in FC group, even better in terms of ROM than in NT group, although VAS and change in distal thigh circumference were comparable in CTC group and in NT group $(p>0.05)$. Fan et $\mathrm{al}^{24}$ also discovered the limited use of a tourniquet in TKA that provides the benefit of decreased limb swelling and better active knee flexion while not compromising the operation time or blood loss and recovery. It therefore seems to be highly desirable to keep the duration of tourniquet to a minimum. ${ }^{25-28}$ The tourniquet application only during cementation may be considered superior, since it hardly influences the functional recovery after TKA.

Some researchers wanted to gather reliable evidence by integrating high-quality clinical trial data. However, their results were not convincing, because the reported influence of the use of tourniquet on the functional outcomes after TKA is variable. Therefore, we assessed several highly objective inflammatory factors such as TNF-a, PTX3, CCL2, SOD1, Mb, and PGE2. Because TNF- $\alpha$, CCL2, interleukin (IL)-6, PTX3, SOD1, PGE2, and Mb, as test indicators, can reflect oxidative stress, inflammatory response, and tissue necrosis ${ }^{12,29-31}$; they are related to quadriceps muscle injury, wound complications, neurovascular injury, swelling and bruising, hidden blood loss, and deep venous thrombosis. No previous studies have assessed the effects of tourniquet application on these inflammatory factors, so the present study not only assessed functional outcomes, but also topical blood samples simultaneously, which can sensitively reflect the degree of local inflammatory reactions and tissue damage to provide insights into pain diagnosis, and serve as indicators to reflect the severity of muscle injury, providing references for clinical treatment.

In topical blood, there were no significant differences statistically in TNF-a, PTX3, CCL2, SOD1, PGE2, and Mb amounts in the three groups at T1, respectively $(p>0.05$,
-Fig. 3), However, the change was lower in CTC and NT groups than in FC group at T2, T3, and T5 (-Fig. 3; - Table 3), confirming that longer tourniquet duration causes much greater inflammation than shorter tourniquet duration and NT in local tissue. A significant finding of the present study was that inflammatory factors in topical tissue, such as TNF-a, PTX3, CCL2, PGE2, SOD1, and Mb, were in positive correlation with tourniquet duration, postoperative early function was related to tourniquet duration as well. The tourniquet, therefore, appears to be a significant source of postoperative pain, swelling, and reduction of ROM. After 24 hours of reperfusion, all inflammatory factors decreased in topical samples. these amounts were closer to preoperative levels in CTC and NT group than those in FC group, suggesting that inflammatory reactions and cell damage are reversible; the shorter the tourniquet duration, the faster the recovery.

In the present trial, the short-duration tourniquet and NT groups were associated with better clinical outcomes, less pain, and reduced limb swelling during the early stage of rehabilitation ( - Fig. 2; - Table 2). Similar results were reported by Zhang et $\mathrm{al}^{19}$ and Ejaz et al. $^{32}$ A possible explanation is that longer tourniquet use results in more pronounced oxidative stress, inflammatory response, and tissue necrosis. TNF- $\alpha$, CCL2, IL- 6 , PTX3, SOD1, PGE2, Mb, and other proinflammatory cytokines are increased, contributing to enhanced telangiectasia and capillary permeability while promoting inflammatory cell infiltration and exudation, followed by severe congestion and acute inflammatory edema. ${ }^{33-40}$ Enhanced swelling also increases soft-tissue tension; additional swelling may hinder the patient's early postoperative rehabilitation exercises. Furthermore, as Dennis et al ${ }^{12}$ reported, patients submitted to TKA using a tourniquet have diminished quadriceps strength. Loss of lower quadriceps strength may result in reduced recovery of the active ROM of the knee. Postoperative pain might be caused by physical damage, reperfusion injury and even fibrotic events in the muscle tissue. ${ }^{23}$ Due to nerve injury, inflammatory reactions and necrotic tissue infection induce various immune and glial cells in the peripheral nerve to 
produce a variety of proinflammatory cytokines and chemokines which cam break the balance between proinflammatory and anti-inflammatory cytokines in the microenvironment, decrease the thresholds of excitability in peripheral and central neurons, increase excitability of neurons and hyperalgesia, and cause pain. ${ }^{12,33-40}$

However, between CTC and NT groups, in term of PTX3, PGE2, and Mb, there was no statistical difference. Surprisingly, in term of TNF- $\alpha$, CCL2, SOD1, better results were observed in CTC group compared with NT group. The results showed that it is not the best without tourniquets, but reasonable application of tourniquet can achieve the best results in TKA.

Besides, in our RCT, intraoperative blood loss was CTC versus FC versus NT $(p<0.01)$, postoperative blood loss was CTC versus FC versus NT $(p<0.01)$, and total blood loss was CTC versus FC versus NT ( $p<0.01$; - Table 11). Blood transfusion was as follows: 5 of 50 patients in FC group, 2 of 50 patients in CTC group, and 14 of 50 patients in NT group. Days to discharge were as follows: CTC versus FC $(p<0.05)$, CTC versus NT $(p<0.05)$, and NT versus FC ( $p>0.05$; - Table 12).

Compared with the other two groups, FC group has the least intraoperative blood loss and the most postoperative blood loss (-Table 11). This finding is in line with the findings of many other previous authors. Due to compression of vessels of lower limb by tourniquet, the blood flow distal to tourniquet is reduced, and therefore the blood loss is lesser. It was believe that with the use of tourniquet, a bloodless operating field is created which helps in better visualization and requires less operative time. However, once the tourniquet is deflated at the end of the procedure, there is a reactionary increase in the blood flow to that limb, due to which there is comparatively more blood loss in the drain in long-duration tourniquet group, as compared with short-duration and NT group.

CTC group has the least postoperative and total blood loss, with the use of tourniquet, a bloodless operating field is created which helps in better visualization and requires less operative time, while not increasing the number of transfusion and operation time. In addition, compared with long-duration tourniquet application, the limited use of a tourniquet results in less knee pain, less time needed to achieve SLR, and less minor complications following TKA. Correspondingly, the functional recovery seems to be faster during the early rehabilitation period and the hospital stay period was significantly reduced.

NT group has the most intraoperative and total blood loss, a blood operating field made worse visualization and requires more operative time, besides increasing the number of transfusion and extending hospital stay.

By comparing with each other group, the present study, through a combination of inflammatory marker changes in blood and clinical manifestations drew a reliable conclusion: patients in CTC group have more advantages than in FC group and in NT group for faster recovery postoperatively. Reasonable application of tourniquet can effectively reduce ischemiareperfusion injury and the amounts of inflammatory factors, can significantly decrease swelling and pain, can promote functional exercise in the early stage, and can speed up recovery.
We acknowledge that the detrimental effect of the tourniquet is time dependent, ${ }^{6}$ there are still controversies on the optimal timing of tourniquet application. Currently, instead of discussing, whether or not to use a tourniquet in TKA, we suggest refining the debate to focus on the acceptable duration of tourniquet. Future research into optimal tourniquet time would determine the ideal individualized strategy for tourniquet use in TKA, understanding the time-dependent influence of the tourniquet in TKA patients would improve the overall therapeutic outcome and safety.

\section{Limitations}

The main limitation of this study is that it failed to carry out long-term detection of inflammatory markers and postoperative rehabilitation process. Further, large well-designed RCTs with extensive follow-up are still needed to validate this research.

\section{Conclusion}

Reduced amounts of inflammatory factors, decreased limb swelling, less pain, and faster recovery were achieved with short-duration tourniquet use in the initial postoperative rehabilitation period which have showed that short-duration tourniquet use in TKA may be advantageous to patient recovery, without overt detrimental effects. However, optimal tourniquet time are required to clarify for tourniquet use in TKA.

\section{Availability of Data and Materials}

We declare that the materials described in the manuscript, including all relevant raw data, will be freely available to any scientist wishing to use them for noncommercial purposes, without breaching participant confidentiality.

\section{Authors' Contributions}

Q.C., N.B., Z.H., and J.Z. performed the experiments. Q.C. and Y.L. analyzed the data and drafted the manuscript. Q.W. collected the specimens. J.Z., N.B., and J.M. revised the manuscript critically for important intellectual content. All authors read and approved the final manuscript.

\section{Ethical Approval}

Ethics Committee of Jinling Hospital Affiliated to the Medical College of Nanjing University; Clinical Trial Registration number 2018NZKY-004-02. Registered on March 13, 2018. All procedures, involving human participants, were in accordance with the ethical standards of the institutional research committee and the 1964 Helsinki declaration and its amendments or comparable ethical standards. Written informed consent was obtained from all participants.

\section{Funding}

This work was supported by the Natural Science Foundation of Jiangsu Province of China (no.: BK20161385), and Social Development-General Program of Science and 
Technology Department of Jiangsu Province (no.: BE2015686).

\section{Conflict of Interest \\ None declared.}

\section{Acknowledgments}

The authors would like to thank all the participants in this study.

\section{References}

1 Smith TO, Hing CB. Is a tourniquet beneficial in total knee replacement surgery? A meta-analysis and systematic review. Knee 2010;17(02):141-147

2 Li B, Wen Y, Wu H, Qian Q Lin X, Zhao H. The effect of tourniquet use on hidden blood loss in total knee arthroplasty. Int Orthop 2009;33(05):1263-1268

3 Silver R, de la Garza J, Rang M, Koreska J. Limb swelling after release of a tourniquet. Clin Orthop Relat Res 1986;(206):86-89

4 Liu D, Graham D, Gillies K, Gillies RM. Effects of tourniquet use on quadriceps function and pain in total knee arthroplasty. Knee Surg Relat Res 2014;26(04):207-213

5 Hernandez AJ, Almeida AM, Fávaro E, Sguizzato GT. The influence of tourniquet use and operative time on the incidence of deep vein thrombosis in total knee arthroplasty. Clinics (São Paulo) 2012;67 (09):1053-1057

6 Butt U, Ahmad R, Aspros D, Bannister GC. Factors affecting wound ooze in total knee replacement. Ann R Coll Surg Engl 2011;93(01): 54-56

7 Lohmann-Jensen R, Holsgaard-Larsen A, Emmeluth C, Overgaard $S$, Jensen $C$. The efficacy of tourniquet assisted total knee arthroplasty on patient-reported and performance-based physical function: a randomized controlled trial protocol. BMC Musculoskelet Disord 2014;15:110

8 Tie K, Hu D, Qi Y, Wang H, Chen L. Effects of tourniquet release on total knee arthroplasty. Orthopedics 2016;39(04):e642-e650

9 Wang K, Ni S, Li Z, et al. The effects of tourniquet use in total knee arthroplasty: a randomized, controlled trial. Knee Surg Sports Traumatol Arthrosc 2017;25(09):2849-2857

10 Lotke PA, Faralli VJ, Orenstein EM, Ecker ML. Blood loss after total knee replacement. Effects of tourniquet release and continuous passive motion. J Bone Joint Surg Am 1991;73(07): 1037-1040

11 Tai TW, Lin CJ, Jou IM, Chang CW, Lai KA, Yang CY. Tourniquet use in total knee arthroplasty: a meta-analysis. Knee Surg Sports Traumatol Arthrosc 2011;19(07):1121-1130

12 Dennis DA, Kittelson AJ, Yang CC, Miner TM, Kim RH, StevensLapsley JE. Does tourniquet use in TKA affect recovery of lower extremity strength and function? A randomized trial. Clin Orthop Relat Res 2016;474(01):69-77

13 Huang HT, Su JY, Chang JK, Chen CH, Wang GJ. The early clinical outcome of minimally invasive quadriceps-sparing total knee arthroplasty: report of a 2-year follow-up. J Arthroplasty 2007; 22(07):1007-1012

14 Wakai A, Winter DC, Street JT, Redmond PH. Pneumatic tourniquets in extremity surgery. J Am Acad Orthop Surg 2001;9(05): 345-351

15 Clarke MT, Longstaff L, Edwards D, Rushton N. Tourniquet-induced wound hypoxia after total knee replacement. J Bone Joint Surg Br 2001;83(01):40-44

16 Pfitzner T, von Roth P, Voerkelius N, Mayr H, Perka C, Hube R. Influence of the tourniquet on tibial cement mantle thickness in primary total knee arthroplasty. Knee Surg Sports Traumatol Arthrosc 2016;24(01):96-101
17 Rathod P, Deshmukh A, Robinson J, Greiz M, Ranawat A, Rodriguez $\mathrm{J}$. Does tourniquet time in primary total knee arthroplasty inflfluence clinical recovery? J Knee Surg 2015;28(04):335-342

18 Huang ZY, Pei FX, Ma J, et al. Comparison of three different tourniquet application strategies for minimally invasive total knee arthroplasty: a prospective non-randomized clinical trial. Arch Orthop Trauma Surg 2014;134(04):561-570

19 Zhang W, Li N, Chen S, Tan Y, Al-Aidaros M, Chen L. The effects of a tourniquet used in total knee arthroplasty: a meta-analysis. J Orthop Surg Res 2014;9(01):13

20 Stroh DA, Johnson AJ, Mont MA, Bonutti PM. Excellent clinical outcomes in total knee arthroplasty performed without a tourniquet. Surg Technol Int 2011;21:189-193

21 Zhang FJ, Xiao Y, Liu YB, Tian X, Gao ZG. Clinical effects of applying a tourniquet in total knee arthroplasty on blood loss. Chin Med J (Engl) 2010;123(21):3030-3033

$22 \mathrm{Li}$ X, Yin L, Chen ZY, et al. The effect of tourniquet use in total knee arthroplasty: grading the evidence through an updated metaanalysis of randomized, controlled trials. Eur J Orthop Surg Traumatol 2014;24(06):973-986

23 Ledin H, Aspenberg P, Good L. Tourniquet use in total knee replacement does not improve fixation, but appears to reduce final range of motion. Acta Orthop 2012;83(05):499-503

24 Fan Y, Jin J, Sun Z, et al. The limited use of a tourniquet during total knee arthroplasty: a randomized controlled trial. Knee 2014;21 (06):1263-1268

25 Klenerman L, Biswas M, Hulands GH, Rhodes AM. Systemic and local effects of the application of a tourniquet. J Bone Joint Surg Br 1980;62(03):385-388

26 Patterson S, Klenerman L. The effect of pneumatic tourniquets on the ultrastructure of skeletal muscle. J Bone Joint Surg Br 1979; 61-B(02):178-183

27 Erskine JG, Fraser C, Simpson R, Protheroe K, Walker ID. Blood loss with knee joint replacement. J R Coll Surg Edinb 1981;26(05): 295-297

28 Pedowitz RA, Gershuni DH, Schmidt AH, Fridén J, Rydevik BL, Hargens AR. Muscle injury induced beneath and distal to a pneumatic tourniquet: a quantitative animal study of effects of tourniquet pressure and duration. J Hand Surg Am 1991;16(04):610-621

29 Guler O, Mahirogullari M, Isyar M, et al. Comparison of quadriceps muscle volume after unilateral total knee arthroplasty with and without tourniquet use. Knee Surg Sports Traumatol Arthrosc 2016;24(08):2595-2605

30 Chen S, Li J, Peng H, Zhou J, Fang H, Zheng H. The influence of a half-course tourniquet strategy on peri-operative blood loss and early functional recovery in primary total knee arthroplasty. Int Orthop 2014;38(02):355-359

31 Jawhar A, Ponelies N, Schild L. Effect of limited ischemia time on the amount and function of mitochondria within human skeletal muscle cells. Eur J Trauma Emerg Surg 2016;42(06):767-773

32 Ejaz A, Laursen AC, Kappel A, et al. Faster recovery without the use of a tourniquet in total knee arthroplasty. Acta Orthop 2014;85 (04):422-426

33 Bottazzi B, Inforzato A, Messa M, et al. The pentraxins PTX3 and SAP in innate immunity, regulation of inflammation and tissue remodelling. J Hepatol 2016;64(06):1416-1427

34 Feldmann M, Maini RN. Anti-TNF alpha therapy of rheumatoid arthritis: what have we learned? Annu Rev Immunol 2001; 19:163-196

35 Gao FQ, Li ZJ, Zhang K, Huang D, Liu ZJ. Risk factors for lower limb swelling after primary total knee arthroplasty. Chin Med J (Engl) 2011;124(23):3896-3899

36 Liu S, Qu X, Liu F, Wang C. Pentraxin 3 as a prognostic biomarker in patients with systemic inflammation or infection. Mediators Inflamm 2014;2014:421429

37 Wood S, Jayaraman V, Huelsmann EJ, et al. Pro-inflammatory chemokine CCL2 (MCP-1) promotes healing in diabetic wounds 
by restoring the macrophage response. PLoS One 2014;9(03): e91574

38 Xie C, Li X, Wu J, et al. Anti-inflammatory activity of magnesium isoglycyrrhizinate through inhibition of phospholipase A2/arachidonic acid pathway. Inflammation 2015;38(04):1639-1648
39 Yasunaga T, Ikeda S, Koga S, et al. Plasma pentraxin 3 is a more potent predictor of endothelial dysfunction than high-sensitive Creactive protein. Int Heart J 2014;55(02):160-164

40 Zweier JL, Talukder MA. The role of oxidants and free radicals in reperfusion injury. Cardiovasc Res 2006;70(02):181-190 